\title{
PLANETARY SCIENCE MISSION OPERATIONS FOR THE NEXT DECADE AND BEYOND
}

Author

Ralph L. McNutt, Jr.

The Johns Hopkins University Applied Physics Laboratory

240-228-5435

Ralph.McNutt@jhuapl.edu

Co-Authors

W. James Adams (National Aeronautics and Space Administration, retired)

James L. Green (Chief Scientist, National Aeronautics and Space Administration) Gael F. Squibb (Jet Propulsion Laboratory, California Institute of Technology, retired)

\section{Endorsers}

Benjamin K. Malphrus (Lunar Icecube Principal Investigator, Morehead State University)

Laureano A Cangahuala (Europa-Clipper Mission Manager, JPL)

Vinton G Cerf (Distinguished Visiting Scientist, JPL)

Debra Bass (Psyche Mission Manager, JPL)

Arthur Amadore (Mars 2020 Mission Manager, JPL)

Chuck Scott (InSight Project Manager, JPL)

John I. Nagy (MAVEN Mission Manager, GSFC)

Jay Trimble (VIPER Mission Manager, ARC)

Raymond E. Arvidson (James S. McDonnell Distinguished University Professor, Washington University in St. Louis)

Brian P. Duncan (Supervisor, Space Systems Implementation Branch, APL)

Maher F. Hanna (Mars 2020 Ground Data System Manager, JPL)

This document contains pre-decisional information - for planning and discussion purposes only. 


\title{
PLANETARY SCIENCE MISSION OPERATIONS FOR THE NEXT DECADE AND BEYOND
}

\author{
Ralph L. McNutt, Jr., W. James Adams, James L. Green, and Gael F. Squibb
}

\section{Executive Summary}

A successful mission occurs only if there are robust mission operations and data systems on the ground to control, collect and analyze health and safety data, and to process and distribute science data. Therefore, NASA's Planetary Science Division (PSD) has invested strategically in the Advanced Multi-Mission Operations System (AMMOS) in the past two decades to reduce cost and risk for deep-space, robotic missions. AMMOS provides all of the elements of an end-to-end Mission Operations System (MOS) and a Ground Data System (GDS), and it is used extensively across NASA Science Divisions and by international partners.

AMMOS is a solid foundation on which to build the MOS/GDS for the demanding missions of the next decade and beyond, enabling successful science missions and scientific discoveries. Planetary Science will benefit from expanding the use of AMMOS on all future deep space missions by avoiding the development and maintenance of multiple, duplicative ground systems designed, built, and paid for by each individual project. We urge the Committee to support AMMOS in the Decadal Report with the following important specifics:

- As NASA's only dedicated MOS/GDS for deep-space, robotic missions, AMMOS is the natural foundation for developing a seamless operations model from spacecraft control to instrument data delivery to the scientist. Additionally, AMMOS offers continual incorporation of emerging new technologies such as utilization of the "cloud," adoption of machine learning and artificial intelligence (AI), and enabling low-cost MOS/GDS for small missions. An AMMOS end-to-end architecture - navigation and mission design; spacecraft command, control, and analysis; science planning; support of science pipeline automation; and formatting of science data for deposit in the Planetary Data System (PDS) - can serve as the baseline for future system development and technological progress to enhance discovery. Further, cyber-security is an increasingly critical function of MOS with evolving demands. The AMMOS-based, project-like environment with a continual cyber-security focus means the system will remain current and robust, reducing project efforts and costs while keeping up with ever-evolving security requirements.

- Increased adoption of AMMOS for deep space missions will further mission and science robustness, reliability, and cost control. To further this approach, greater scrutiny of MOS/GDS selection and use should be added to mission concept proposals and Concept Study Reports (CSRs). Since the Planetary Science Division (PSD) has invested heavily in AMMOS over the past decades, and with significant mission use, its heritage and robustness are well proven. Coupled with the fact that small missions must utilize every available dollar to maximize scientific return while minimizing risk, there is a compelling case that an available end-to-end operating system that has already being paid for by NASA should be the baseline mission operations-ground data system for deep space missions. Moreover, AMMOS tools are provided at no cost to a mission, AMMOS services and products enable rapid and reliable data delivery to science teams and the PDS, and support for AMMOS products is guaranteed for the life of a mission (e.g., Voyager continues to 
use AMMOS-supported products). Hence, missions that use MOS/GDS elements other than AMMOS need to provide detailed, specific element-by-element justification addressing how their system is an improvement over AMMOS, e.g., improves system reliability, increases available mission funding for instruments/science, ensures system cyber-safety, and is supported throughout the life of the mission, regardless of mission length. Concomitantly, potential PIs would benefit from understanding AMMOS services and products, so we advocate including an AMMOS briefing in PI training activities at each mission Announcement of Opportunity (AO) pre-proposal conference.

\section{Background}

The Advanced Multi-Mission Operations System (AMMOS) is the NASA-sponsored ground system for the unique needs of deep space exploration. Conceived initially at the Jet Propulsion Laboratory (JPL), California Institute of Technology, in the mid-1980s as a common mission operations and data processing toolset (Green 1995; Linick 1996), the first documented use of AMMOS was for the Magellan mission (Linick, 1996). Conceived broadly, the Magellan mission made use of what is now considered the telemetry and command system portion of AMMOS. Following recommendations from the Solar System Exploration Committee, AMMOS was established as a NASA-wide entity in 1985 (Green, 1995). The then-PSD Director (J. Green) expanded the AMMOS charter in 2008 to make it available, without charge, to all other, then-existing NASA Science Divisions (Astrophysics, Earth Science, and Heliophysics).

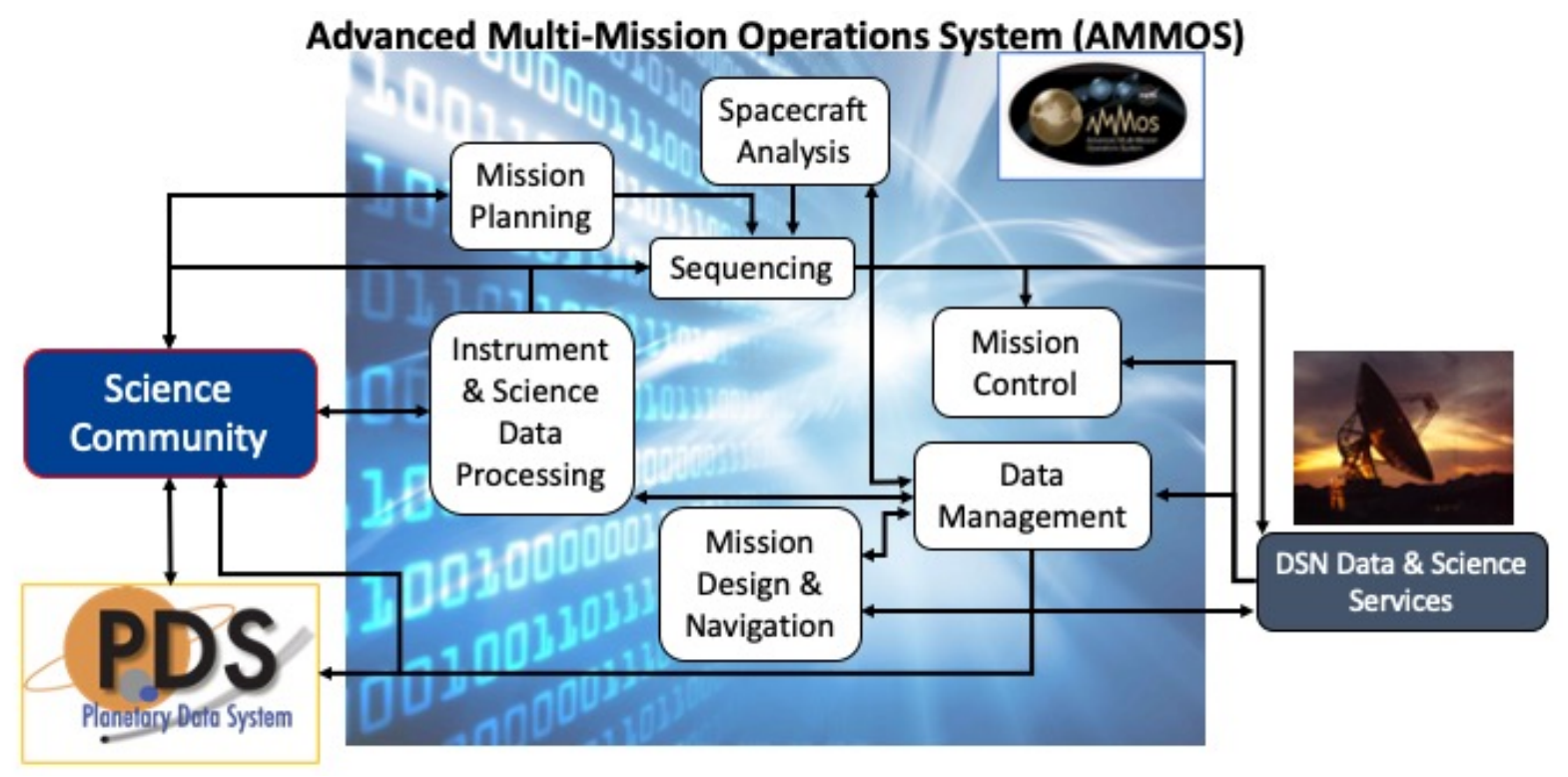

As seen in the diagram above, today AMMOS provides a set of services and products across all MOS/GDS elements. These are designed to enable formulation and implementation of a ground system, from initial mission development to planning spacecraft operations to processing of telemetry received by the Deep Space Network (DSN) to delivery of data to the PDS. The AMMOS elements, which support these activities, are Mission Planning, Sequencing and Analysis; Multi-Mission 
Control Systems; Mission Design and Navigation; and Instrument Data Systems and Archiving; as well as cross-cutting services (e.g., configuration management; process automation; cybersecurity;

and multi-mission integration, test, and deployment) and specialized services (e.g., Relay Operations for Mars and DSN Scheduling). These services and products are guaranteed to be maintained for the life of a NASA mission, and the products themselves are provided at no cost to the mission.
Table 1. NASA's Advanced Multi-Mission Operating System (AMMOS) has substantial heritage and robustness

\begin{tabular}{|l|c|}
\hline Current User Base & 62 missions \\
\hline Code Base & $\begin{array}{c}\text { Over } 13 \text { million } \\
\text { software lines of code }\end{array}$ \\
\hline $\begin{array}{l}\text { Annual Operations, } \\
\text { Development, } \\
\text { Maintenance }\end{array}$ & $\begin{array}{l}\text { Staffed as a project, } \\
\text { ensuring continuity }\end{array}$ \\
\hline $\begin{array}{l}\text { Operations \& } \\
\text { Development }\end{array}$ & 25 years \\
\hline
\end{tabular}

Table 1 and Figure 1 describe the heritage and wide-spread deployment of AMMOS. In addition to all Planetary Science missions, five Astrophysics missions, nine Earth Science missions, nine Heliophysics missions, and a small number of missions for other Government agencies make use of some AMMOS services and products. These data include not only NASA-led missions but missions led by the European Space Agency (ESA), the Japanese Aerospace Exploration Agency (JAXA), the Indian Space Research Organization (ISRO), the Korean Aerospace Research Institute (KARI), and the United Arab Emirates Space Agency.

Not only has the scope of AMMOS expanded as mission needs have increased, but also its

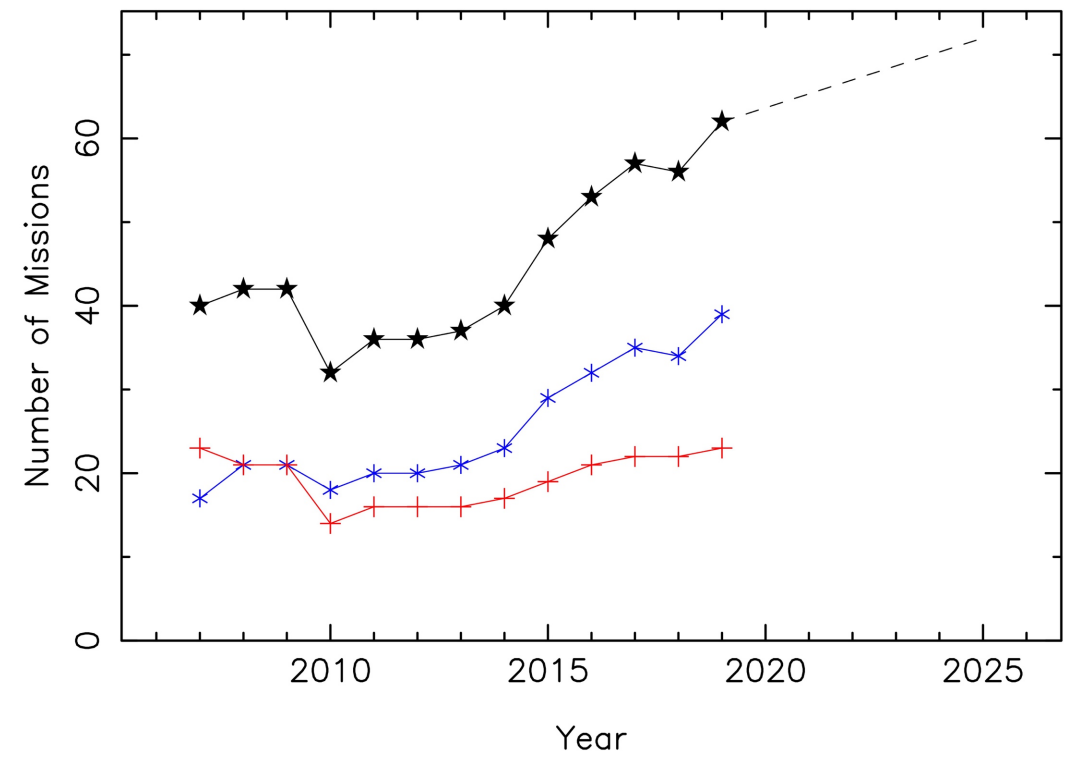

Figure 1. Missions have been increasingly adopting AMMOS services and tools, most notably smaller missions in the recent past. The blue stars (*) show usage by Planetary Science missions, the red crosses $(+)$ show usage by missions from other NASA Science Divisions or human spaceflight, and the black stars ( $\star$ ) show the total usage. The dotted line shows current projection of continued growth based on missions currently in review or formulation. development and implementation base has expanded. Today, both development and implementation are conducted by software engineers across NASA Centers (Ames Research Center [ARC], Goddard Space Flight Center [GSFC], Katherine Johnson Independent Verification \& Validation Facility, Marshall Space Flight Center [MSFC]), other Government agencies (U.S. Geological Survey [USGS]), University Affiliated Research Centers (UARCs) (Johns Hopkins University Applied Physics Laboratory [APL]), Federally Funded Research and Development Centers (FFRDCs) (JPL, California Institute of Technology), numerous universities, and international entities. AMMOS has a Project Users Group (PUG) that meets 
regularly to ensure the systems, products, and services remain responsive to the science goals and objectives of missions and that the AMMOS long-term development and maintenance plans are responsive to mission needs. The PUG is open to all users of AMMOS products and services, regardless of extent of use.

The AMMOS Program also conducts regular calls for technology development, guided by a technology roadmap developed with input from the PUG. These proposal calls are open to all members of the Planetary Science community, and provide support from which scientists and engineers can draw to design and develop future capabilities. The most recent such call was in December 2019.

\section{Challenges and Opportunities for the Next Decade and Beyond}

Since the Vision and Voyages Planetary Decadal Survey (National Research Council, 2011), the landscape for ground systems has changed significantly. Computing capability and capacity have risen dramatically. The emergence of low-cost, commercial space systems and small spacecraft and the ability of the latter to contribute to "decadal class" science has created a broader pool of mission Principal Investigators (PIs) and capable mission prime contractors. The use of state-of-the-art, cloud-based techniques for computing and storage is now expected. Crowd and citizen science programs also mean a demand for more open access in real time to spacecraft, systems, and data. The increased reliance on spacecraft autonomy and the emergence of machine learning and AI represent significant maintenance and evolution stressors for existing flight and ground data systems. Overall these changes are a good environment for planetary science, but it also means that a variety of MOS/GDSs are being developed with a wide range of risk, and some with questionable pedigree. AMMOS's architecture, availability, cost, and program-like structure creates stability and focus and places it in the perfect position to provide a cost-effective and low-risk system for future missions in key areas, such as the following:

Surface Assets and Aerial Vehicle Operations Planetary exploration has followed a general pattern of fly by, orbit, land, rove, and return samples. Over the next decade, in addition to multiple landers and rovers on the surface of Mars, there will be aerial vehicles in the atmospheres of Mars and Titan and potentially other solar system bodies. An exciting development in the design of Planetary Science missions is the use of multiple elements in a collaborative fashion, for which the first example will be the Mars 2020 Perseverance rover and the Mars Helicopter Scout Ingenuity; similar multi-element collaborative studies are envisioned a variety of mission concepts (e.g., the Venus Flagship Mission concept, one of the Planetary Mission Concept Studies). The use of multiple coordinated assets will allow for new science investigations, but realizing these possibilities will demand new levels of surface operations, mobility, navigation, and inter-platform coordination. New and unique requirements and tools will emerge for MOS/GDSs, and the Planetary Science community will gain the most benefit if these new tools are both multi-mission and scalable, capable of being used or adapted to other scenarios with varying numbers of assets or vehicles. AMMOS already provides a substantial set of products and services for surface navigation, surface operations, and science data return, ranging from mission control to surface activity planning and execution to on-board science data processing and science data delivery to the science team to archiving in the PDS. It is therefore a natural foundation for building the next set of more capable surface missions. 
Autonomy Missions currently incorporate some level of autonomous operations, but typically only for short, but intensive, durations that are often dictated by light-time delay (e.g., Mars entry, descent, and landing; New Horizons transit through the Pluto-Charon system). AMMOS already provides products for on-board autonomous science data processing, such as Autonomous Exploration for Gathering Increased Science (AEGIS) on the Curiosity rover. Answering future science questions may well demand increasing levels of autonomous operations for longer durations, with potential examples including operations in planetary atmospheres (Dragonfly or long-duration balloons at Venus); exploration of lunar or Martian caves or ice crevasses on an Ocean World; surface operations during loss of contact with the Earth (e.g., the Planetary Mission Concept Studies Mercury Lander concept involves multi-week operations with no Earth contact) or, perhaps the ultimate example, a submersible vehicle in an Ocean World ocean. These examples, or other potential scenarios, also place new demands on the ground system. The ground system will need to be able to specify operations in manners that are more robust and resilient than current, highly-sequenced operations. In addition, once contact is re-established with a spacecraft, the ground system and mission operations team will have to understand what operations the spacecraft undertook, particularly if there were deviations from what was intended. AMMOS already is developing the capabilities to enable mission operations teams to operate effectively the autonomous spacecraft of the future.

Relays and Networking The concept of interplanetary relays and networks could enable missions elsewhere across the Solar System, beyond the current instance at Mars, with a lunar communication network being a possible requirement as lunar exploration expands and Artemis comes to reality, carrying scientific instruments on human-scale missions. Other possibilities also exist, with one example being elements of a Venus Flagship Mission. NASA/AMMOS operates, in collaboration with ESA, the Mars Relay Network, which has been a critical element of the success of all Martian surface assets, with some landers relying exclusively on the Mars Relay Network for return of science data. Therefore, AMMOS presents a natural foundation, yet there is the substantial risk that multiple developments will occur leading to unnecessary costs, a lack of inter-operability, and difficulties in returning science data. Further, AMMOS is also investigating incorporation of elements of delay/disruption tolerant networking (DTN), which can be a significant challenge to commercial operations tools.

Constellations Many mission concepts within the Planetary Mission Concept Studies (e.g., Venus Flagship Mission concept and Mars Orbiters for Surface-Atmosphere-Ionosphere Connections [MOSAIC]) and multiple mission concept white papers envision coordinated observations of other planets. Operating multiple spacecraft in a coordinated fashion is now common for Earth Science and Heliophysics missions. Developing or incorporating efficient means for operating a constellation of spacecraft at a planetary target likely will require new services and tools, but also the opportunity to collaborate across NASA Science Divisions, across NASA Centers and universities, and, potentially, with industry. AMMOS already works with constellations in the Heliophysics Division and is working on multi-mission products for future Planetary Science missions, alleviating the technology development for missions that utilize non-AMMOS MOS/GDS.

Compute Environments The environment for software development and computing has changed dramatically, and will continue to do so, as open sourcing, crowd participation, cloud operations, and adaptive autonomy technologies like machine learning and AI become commonplace and reliable and are eventually integrated into operations. These evolutions offer numerous benefits, 
such as (1) wider participation in software development as more code becomes open source; (2) automated trend analysis of telemetry data and early identification of anomalies or out-offamily events; and (3) rapid, on-demand processing of data from a surface asset (rover, aerial vehicle, and/or others) for operations planning via provision of cloud computing. AMMOS can serve as the framework around which to structure these improvements in mission operations, naturally evolving to incorporate these technologies and advances to enable increased and more reliable science return.

Multi-Organization Partnerships There has been a long and laudable history of collaboration within Planetary Science, with multi-mission partnerships among NASA Centers (ARC, GSFC, Johnson Independent Verification \& Validation Facility, Johnson Space Center, MSFC), other Government agencies (USGS), FFRDCs (JPL), UARCs (APL), numerous universities, and private industry, in addition to mission partnerships with the ESA and JAXA. Further, many new actors are rapidly emerging and creating new partnerships that are not always mission-based, including ISRO, the United Arab Emirates Space Agency, and KARI. The next decade will also see the emergence of not-for-profit or philanthropic entities such as U.S. universities sponsoring planetary science spacecraft and commercial entities operating on and around the Moon, for which AMMOS's flexibility, existing products, and open-source strategy are well suited. In order to ensure maximum interoperability for NASA missions and science instruments, AMMOS developers participate in international standards bodies such as the Collaborative Committee for Space Data Systems (CCSDS), while simultaneously respecting U.S. Government laws (notably International Traffic in Arms Regulations [ITAR]).

Programmatic Importance As more nations are developing space missions to planetary destinations, along with commercial partners, there is a natural need for those entities to work with NASA because they need capabilities that AMMOS provides, such as planetary navigation and ephemerides. From a PSD perspective, the AMMOS software has enabled productive bilateral negotiations in which PSD has offered AMMOS capabilities and obtained, through the fundamental quid pro quo process, important considerations such as an opportunity to provide an instrument or obtain critical mission data from those non-NASA missions. The benefits of this "bargaining chip" should not be underestimated, particularly as more multi-organizational partnerships emerge in the future.

Cybersecurity An unfortunate reality is that cybersecurity threats have become more sophisticated and widespread since the Vision and Voyages report and will continue to evolve. NASA's missions are high profile and high value and represent tempting targets, particularly should they be perceived as "softer" than other U.S. Government missions. It is possible that the coming decade will see a rise in attempts to interfere with the control and data systems crucial to mission operations, and the U.S. Government has begun to require encryption of commanding sequences. AMMOS already provides NASA-compliant cybersecurity as part of its services and products and provides a ready means for ensuring compliance with current and future NASA and U.S. Government requirements. This need to ensure cybersecurity will require AMMOS to implement and maintain systems that enable the integration of processes and information across the full breadth of partner organizations while achieving a risk-based balance between the historical level of interaction in an open environment along with inter-operability, technology infusion, system functionality and operability, and security. While a challenge, there will also be opportunities for collaboration with universities and, most notably, industries, e.g. as is currently emerging with the Commercial Lunar Payload Services (CLPS) program. Therefore, 
use of AMMOS can relieve a mission from significant cost and technical burdens of keeping up with NASA cyber-security requirements and updates.

\section{Summary}

Operating the ambitious and challenging portfolio of deep-space missions in the next decade and beyond while continuing to ensure successful operations of existing and ongoing missions, requires taking advantage of the decades of experience in mission operations and science data management embodied within the Advanced Multi-Mission Operations System (AMMOS). With extensively tested, high-heritage services and tools providing all of the elements of mission operations and data processing, AMMOS reduces the risk of future missions and enables cost savings across NASA/PSD. We encourage the Decadal Panel to support expanded use of AMMOS across the Planetary Mission spectrum in order to ensure secure, reliable, time-tested mission operations-ground data systems that will reduce mission cost and increase science opportunities as the next decade of planetary exploration becomes a reality.

\section{References}

Green, W. B. 1995, "Multimission Ground Data System Support for NASA's Planetary Program," Acta Astronautica, 37, 407; doi: 10.1016/0094-5765(95)00067-A.

Linick, T. D. 1996, "Multimission Operations Systems - A Management Perspective," AIAA Paper 96-4421, AIAA Space Programs and Technologies Conference, Huntsville, AL; doi: $10.2514 / 6.1996-4421$.

National Research Council. 2011. Vision and Voyages for Planetary Science in the Decade 20132022. Washington, DC: The National Academies Press. https://doi.org/10.17226/13117. 